\title{
An Algerian Family with TNF Receptor Associated Periodic Syndrome (TRAPS) Associated Amyloidosis and the p.Thr79Met Mutation
}

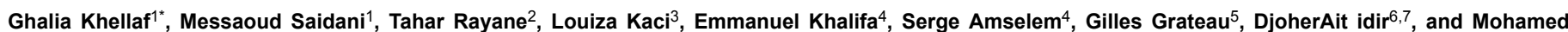
Benabadji ${ }^{1}$

${ }^{1}$ Department of Nephrology, University of Algiers Benkheda Youssef, Beni Messous Hospital, Algeria

${ }^{2}$ Department of Nephrology, University of Algiers Benkheda Youssef, Nefissa Hamoud Hospital, Algeria

${ }^{3}$ Pathology Private Laboratory, Algeria

${ }^{4}$ Department of Molecular Genetics, Sorbonne University, Armand Trousseau Hospital, Paris, France

${ }^{5}$ Department of Internal Medicine, Sorbonne University, Tenon Hospital, Paris, France

${ }^{6}$ Faculty of Biological Sciences, University of Science and Technology - Houari Boumediene, Algeria

${ }^{7}$ Faculty of Sciences, M'Hamed Bouguerra University, Boumerdes, Algeria

"Corresponding author: Ghalia Khellaf, Department of Nephrology, University of Algiers Benkheda Youssef, Beni Messous Hospital, Algeria, Tel: 0773641197; E-mail: g.khellaf@yahoo.fr

Received Date: June 18, 2018; Accepted Date: July 09, 2018; Published Date: July 12, 2018

Copyright: (C) 2018 Khellaf G, et al. This is an open-access article distributed under the terms of the Creative Commons Attribution License, which permits unrestricted use, distribution, and reproduction in any medium, provided the original author and source are credited.

\begin{abstract}
We report the case of a 36-year-old woman of Algerian descent on dialysis for renal AA amyloidosis. She reported since the age of 6 years recurrent episodes of 2 weeks duration characterized by fever, weakness, abdominal pain, migrating myalgias, arthralgias, erythema, and chest pain. Diagnosis of TRAPS was confirmed by the presence of heterozygous $p$.Thr79Met mutation in TNFRSF1A. Genetic evaluation of all affected members of her family disclosed the p.Thr79Met mutation in TNFRSF1A, including one patient with amyloidosis. Genotypephenotype correlations revealed variable clinical presentation and incomplete penetrance. TRAPS is a rare Mendelian auto-inflammatory disease that may be observed in populations where familial Mediterranean fever is highly prevalent. In this context, a high level of clinical suspicion is mandatory to evoke the diagnosis of TRAPS and initiate the appropriate treatment in order to prevent $A A$ amyloidosis and its renal consequences.
\end{abstract}

Keywords: TRAPS; Algeria; FMF; AA Amyloidosis

\section{Abbreviations}

CRP: C-Reactive Protein; FMF: Familial Mediterranean Fever; IL: Interleukin; MEVF: Mediterranean Fever; SAA: Serum Amyloid A Protein; TNFRSF1A: (TNF) Tumor Necrosis Factor Receptor Super Family 1A; TRAPS: (TNF) Receptor Associated Periodic Syndrome

\section{Introduction}

TNF Receptor Associated Periodic Syndrome (TRAPS) is characterized by recurrent attacks of fever associated with abdominal pain, myalgias, migratory erythematous skin rash, conjunctivitis, and periorbital edema [1]. In contrast to patients with familial Mediterranean fever (FMF), patients with TRAPS usually have a poor response to colchicine, but a favourable response to corticosteroids and to interleukin (IL)-1 inhibitors. TRAPS has been described mostly in families of north European descent. The most thoroughly characterized was a large pedigree of Irish and Scottish ancestry ascertained in Nottingham, England, whose illness was denoted familial Hibernian fever [2]. TRAPS is a very rare disease with an estimated prevalence of about one per million [3]. It has rarely been reported in Arabic population, where FMF is in contrast highly prevalent. We report the first cases of TRAPS complicated by amyloidosis in a family from the south of Algeria, which has been diagnosed late in the course of the disease.

\section{Case Description}

The proband (II/7 Figure 1) is a 36-year-old woman native of southern Algeria. Her parents were first cousins. She was hospitalized in February 2013 for kidney biopsy in front of nephrotic syndrome and renal insufficiency (creatinine clearance at $45 \mathrm{~mL} / \mathrm{min}$ ). She has a history of recurrent inflammatory attacks since the age of 6 years. These attacks are characterized by fever of more than 15 days and fasciitis affecting the limbs with a typical migrating proximal to acral evolutive pattern during the attack. Other symptoms during attacks are abdominal pain first localized then diffuse at the acme of the fever, arthralgias with no signs of arthritis, headache, periorbital edema, and fatigue. She underwent an appendicectomy at the age of 10 that did not suppress recurrent abdominal attacks. Some attacks were triggered by physical stress and menstruation. At the age of 35 years, the patient presented with a major edematous syndrome and orthostatic hypotension, proteinuria at $3 \mathrm{~g} /$ day and hypoalbuminemia at $14 \mathrm{~g} / \mathrm{L}$. 
Citation: Khellaf G, Saidani M, Rayane T, Kaci L, Khalifa E, et al. (2018) An Algerian Family with TNF Receptor Associated Periodic Syndrome (TRAPS) Associated Amyloidosis and the p.Thr79Met Mutation. J Nephrol Ther 8: 314. doi:10.4172/2161-0959.1000314

Page 2 of 4

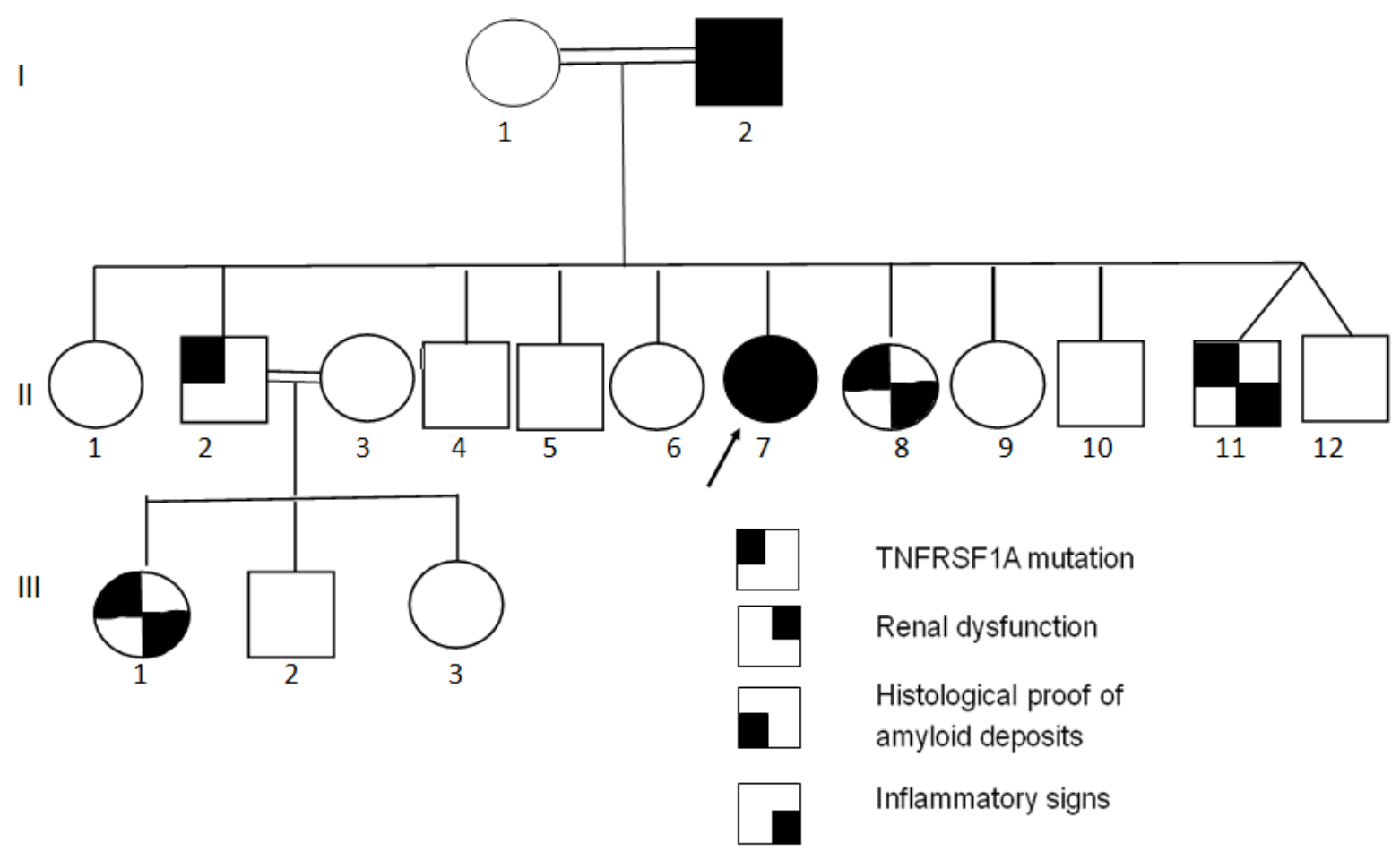

Figure 1: Pedigree of an Arabic Algerian family with TRAPS and AA amyloidosis. The p.Thr79Met mutation is present in Patients (I/2, II/2, $\mathrm{II} / 7, \mathrm{II} / 8, \mathrm{II} / 11$ and III/1).

Inflammation was present with C-reactive protein (CRP) at 120 $\mathrm{mg} / \mathrm{L}$ and anemia (hemoglobin $9 \mathrm{~g} / \mathrm{dL}$ ). Immunological workup was unremarkable for autoimmune diseases (normal complement, $\mathrm{C} 3, \mathrm{C} 4$, negative anti-nuclear antibodies, anti-DNA and anti-neutrophil cytoplasm antibodies), while serum protein electrophoresis and immunofixation excluded gammopathies. Kidney biopsy disclosed AA amyloidosis (Figure 2).

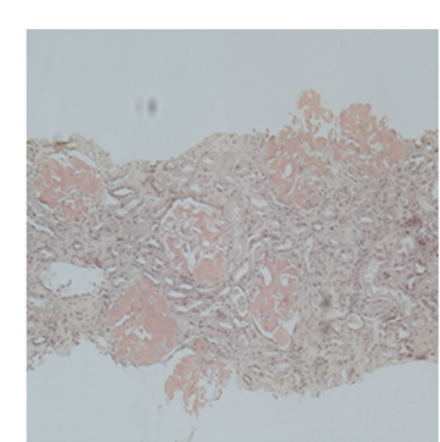

(A)

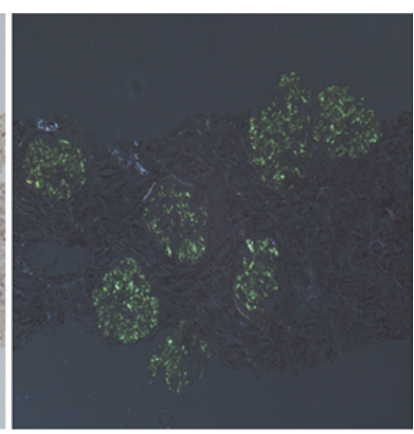

(B)

Figure 2: Renal biopsy of Patient II 7 in Figure 1: (A) Congo red; (B) Congo red under polarized light. Congo red stain disclosed amyloid deposits in the glomeruli mainly in the mesangium and in an interlobular artery.
Finally genetic study was done after informed consent obtained from the patient and all of her family (Figure 1) that showed no mutation in the MEVF gene and disclosed the p.Thr79Met (formerly T50M) mutation in the TNFRSF1A gene at the heterozygote state. Since August 2013, the patient has been on chronic hemodialysis with many complications: hypotension, recurrent thrombosis of the fistula. Attacks are less frequent, spaced with one each six months, but remain very painful especially when fasciitis occurs on the side of the fistula with hyperleukocytosis, thrombocytosis and CRP at $130 \mathrm{mg} / \mathrm{L}$. Attacks remain responsive to intravenous corticosteroid therapy. Amyloidosis is still evolutive as the patient has developed a probable amyloid goitre.

Her father, (Patient I/2 Figure 1) aged 70 years is on hemodialysis since the age of 52 for chronic renal disease of undetermined cause. He had a long history of recurrent attacks since the age of 12 years with approximately 3 attacks per year. Attacks last 15 days with fever, fasciitis, arthralgias, abdominal pain, unilateral scrotitis, periorbital edema. Edematous syndrome appeared at the age of 38 years and hemodialysis was started at the age of 40 . Since he has been on hemodialysis, the patient has remained free of clinical inflammatory attacks; fistula performed at the left radial artery 30 years ago is still functional. He has developed a euthyroid goiter, and his CRP remains normal. Salivary gland biopsy returned in favor of AA amyloidosis (Figure 3). 


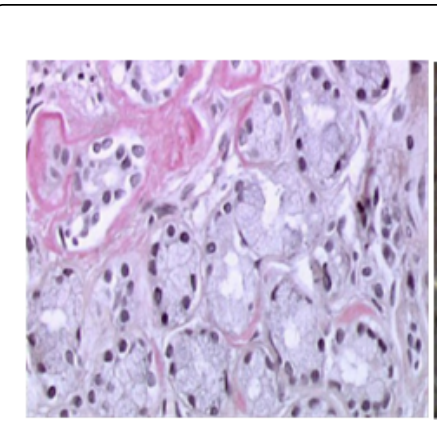

(A)

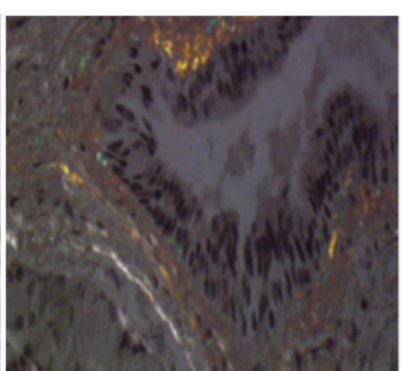

(B)

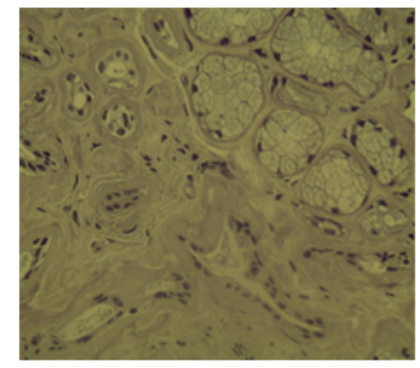

(C)

Figure 3: (A) Congo red; (B) Congo red under polarized light; (C) Immunohistochemistry with anti-SAA antibody (peroxidase method). Amyloid deposits are observed that are strongly marked with anti-SAA antibody.

Genetic study showed no mutation in the MEVF gene and disclosed the same p.Thr79Met mutation in the TNFRSF1A at the heterozygote state.

A sister, (Patient II/8 Figure 1) is a 34-year-old patient who presented with the same symptoms as her elder sister since age 6 , with a periodic biannual attack of 2 weeks: fever, fasciitis, arthralgias, skin lesions, appendectomy at the age of 20, but without renal involvement. Salivary gland biopsy showed no abnormalities. Colchicine was started gradually up to $3 \mathrm{mg} /$ day for one year without any clinico-biological improvement. Genetic study disclosed the same p.Thr79Met mutation in TNFRSF1A at the heterozygote state. Since 2015 the patient is under $10 \mathrm{mg} /$ day of corticosteroids and remains free of symptoms of TRAPS. Her CRP remains slightly elevated between 7 and $10 \mathrm{mg} / \mathrm{L}$.

A brother (Patient II/11 Figure 1) aged 30 had similar symptoms in the pediatric age, with a periodic biannual attack of 2 weeks: arthralgias of the large joints, scrotitis and migrating skin lesions of the limbs. Colchicine was inefficient to prevent attack's recurrences. He has no overt clinical renal disease and salivary gland biopsy showed no amyloid deposits. No mutation was found in MEFV and further study disclosed the p.Thr79Met mutation at the heterozygote state in TNFRSF1A. Since 2015 the patient is treated with prednisone 10 $\mathrm{mg} /$ day and has no clinical symptoms. His CRP is measured at $7 \mathrm{mg} / \mathrm{L}$ maximum. His twin (II/12) is also heterozygous for the p.Thr79Met mutation and is still healthy.

Another brother (II/2 Figure 1) is an asymptomatic man aged 40 in good general condition with no specific history of inflammatory attacks and no signs of renal disease. His daughter is a girl aged 15 (Patient III/1 Figure 1) with a history, since age 5 of quarterly inflammatory attacks of fever, arthralgias, and skin lesions. The patient had several hospitalizations in pediatric wards and the diagnosis of untagged rheumatism was made and symptomatic treatment was started. She was depressed because of prolonged bed rest. In 2014 she had hemoglobin at $10 \mathrm{~g} / \mathrm{dL}$, a CRP at $20 \mathrm{mg} / \mathrm{l}$ and no renal disease. Salivary gland showed no amyloid deposits. She was first treated with colchicine at $2 \mathrm{mg} /$ day without clinical or biological benefit and after genetic results with prednisone at $10 \mathrm{mg} /$ day. She is since clinically asymptomatic with a CRP at $10 \mathrm{mg} / \mathrm{L}$. Both this patient and her father harbor the p.Thr79Met mutation of the TNFRSF1A gene.

\section{Discussion}

We report a TRAPS family native to the south of Algeria. Clinical presentation is quite typical of TRAPS phenotype with quarterly or even semi-annual inflammatory attacks of 15 to 21 days. Symptoms observed during attacks are: migratory fasciitis, arthralgias of large joints, abdominal pain simulating a surgical emergency, unilateral vaginalitis, with a frank acute phase response, all spontaneously resolving in 15 to 28 days [4-6]. The p.Thr59Met mutation found in this family has already been described in TRAPS patients belonging to other populations [1]. Moreover, in our family, it segregates with the disease as all clinically affected individuals over 3 genereations harbour the mutation. However, two individuals harbour this mutation and remain currently free of clinical symptoms, thus conveying the incomplete penetrance of the mutation in this family. Clinical expression is relatively similar in all patients except for amyloidosis that occurred in two of them and at a young age in the proband. Amyloidosis is usually associated with mutations involving cysteine residues that confer the most severe phenotype in TRAPS. However, amyloidosis has already been reported with p.Thr59Met $[5,6]$. These two characteristics: incomplete penetrance and variable expression of the disease including the presence of amyloidosis have widely been described in TRAPS [5,7].

The p.Thr79Met mutation of the TNFRSF1A gene is one of the most frequent associated with TRAPS. It has been described in a number of families, mainly of European ancestry [1]. To our knowledge it has not been described in patients of Algerian origin. Several cases of TRAPS patients have already been reported among the Arab population from the Middle East (Iraq, Kuwait) and Maghreb (Mauritania) and in Africans too, with other mutations (p.Thr66Ile, p.Cys72Tyr, p.Thr79Lys) [8].

We wish to emphasize the delayed diagnosis of TRAPS in this family. Indeed, in the settings of a history of recurrent inflammatory attacks and amyloidosis in a family belonging to a population where FMF is frequent, this latter diagnosis was suspected and colchicine was thus given to the patients. The mode of inheritance of the disease in this family with consanguinity is compatible with apseudo-dominant mode of inheritance. Consanguinity is frequent in this population and favours the emergence of recessive diseases such as FMF. In a second step, the lack of efficacy of colchicine, the absence of MEFV mutations and a more thorough analysis of the symptoms led to the hypothesis of TRAPS in this family. The discovery of a mutation in the TNFRSF1A gene confirmed the diagnosis that allowed a more accurate treatment for the patient's attacks who were sensitive to steroids.

\section{Conclusion}

This family from southern Algeria has a long history of diagnostic wandering. The strong consanguinity in this family is a deceptive 
Citation: Khellaf G, Saidani M, Rayane T, Kaci L, Khalifa E, et al. (2018) An Algerian Family with TNF Receptor Associated Periodic Syndrome (TRAPS) Associated Amyloidosis and the p.Thr79Met Mutation. J Nephrol Ther 8: 314. doi:10.4172/2161-0959.1000314

Page 4 of 4

element since it reinforced the hypothesis of a recessive disease led to the wrong diagnosis of FMF and delayed the diagnosis of TRAPS. This emphasizes the importance of a precise analysis of the clinical symptoms in a context of recurrent familial fever in a country where FMF is highly prevalent as well as the interest of the negative therapeutic test with colchicine in this context. This case also highlights the value of the molecular diagnosis which led to a targeted therapeutic management.

\section{Conflict of Interest}

The authors have no conflict of interest to declare.

\section{Consent Obtained}

All patients and healthy family members gave written consent for genetic analysis and anonymous publication.

\section{Ethical Approval for this Study}

All the patients of this study have given writing consent formal consent is not required. This article does not contain any studies with humans or animals performed by any of the authors.

\section{References}

1. McDermott MF, Aksentijevich I, Galon J, McDermott EM, Ogunkolade BW, et al. (1999) Germline mutations in the extracellular domains of the
55K Da TNF receptor, TNFR1, define a family of dominantly inherited autoinflammatory syndromes. Cell 97: 133-144.

2. Williamson LM, Hull D, Mehta R, Reeves WG, Robinson BH, et al. (1982) Familial Hibernian fever. Q J Med 51: 469-480.

3. Lainka E, Neudorf U, Lohse P, Timmann C, Stojanov S, et al. (2009) Incidence of TNFRSF1A mutations in Germanchildren: epidemiological, clinical and genetic characteristics. Rheumatology (Oxford) 48: 987-991.

4. Hull KM, Drewe E, Aksentijevich I, Singh HK, Wong K, et al. (2002) The TNF receptor-associated periodic syndrome (TRAPS): emerging concepts of an autoinflammatory disorder. Medicine (Baltimore) 81: 349-368.

5. Dodé C, André M, Bienvenu T, Hausfater P, Pêcheux C, et al. (2002) The enlarging clinical, genetic, and population spectrum of tumor necrosis factor receptor-associated periodic syndrome. Arthritis Rheum 46: 2181-2188.

6. Aksentijevich I, Galon J, Soares M, Mansfield E, Hull K, et al. (2001) The Tumor necrosis factor receptor-associated periodic syndrome: new mutations in TNFRSF1A, ancestral origins, genotype-phenotype studies, and evidence for further genetic heterogeneity of periodic fevers. Am J Hum Genet 69: 301-314.

7. Aganna E, Hammond L, Hawkins PN, Aldea A, McKee SA, et al. (2003) Heterogeneity among patients with tumor necrosis factor receptorassociated periodic syndrome phenotypes. Arthritis Rheum 48: 2632-2644.

8. Lachmann HJ, Papa R, Gerhold K, Obici L, Touitou I, et al. (2014) The phenotype of TNF receptor-associated autoinflammatory syndrome (TRAPS) at presentation: a series of 158 cases from the Eurofever/ EUROTRAPS international registry. Ann Rheum Dis 73: 2160-2167. 